\title{
Research of Medical English Paper Translation Under the Skopos Theory
}

\author{
Chen Chen \\ Center for Medical Language and Culture Studies, X'an Medical University, Xi'an 710021, China \\ dsgkevin@163.com
}

\begin{abstract}
With the rapid development of the global economy, Medical English is being used by more and more people in clinical, research, teaching and medical services, as international medical and health cooperation is increasingly strengthened. In order to promote the development of medical research in China and narrow the gap between China and developed countries, researchers and clinicians often need to refer to a large number of Medical English Research Paper in their work. These English papers need to be translated into Chinese for reference. Medical English translation is becoming more and more important. Nowadays, there are many difficulties in the process of the vigorous development of China's medical and health services, including many difficulties in translating English medical works and papers. From the perspective of linguistics and according to the nature of translation, Nida puts forward the famous theory of functional equivalence, which aims to make the conversion between source language and target language normative and reduce differences. Therefore, in the process of medical English translation, in addition to the necessary language foundation and medical professional knowledge, profound translation theory foundation and solid translation skills can also make the translator's translation accurate in content and standardized in language expression. Guided by the definition of functional equivalence, the core concept of Nida's theory, this paper studies the functional equivalence of words, sentences, texts and styles in Medical English Research Paper translation from the perspective of functional teleology.
\end{abstract}

Keywords: Skopos theory; Medical English Research Paper; Translation strategies.

\section{Introduction}

With the goal to put forward the progress of medical study in domestic as well as shorten the gap between China and developed countries, researchers and clinicians often need to consult a large number of medical research papers written in English in Chinese and western countries. Literature sometimes needs to be translated into Chinese for reference at any time. Medical English translation is becoming more and more important. Nowadays, there are many difficulties in the process of the vigorous development of China's medical and health undertakings, including the difficulties in translating medical treatises written in English. Seen from the point of linguistics as well as taking the nature of translation into consideration, a famous translation theory has been proposed, named of "dynamic equivalence", at the same time, it was named "functional equivalence". Medical English must have a large number of terms, vocabulary data, long sentences and complex structure. Thus, while taking medical English translation, it is essential to pay attention to the basic linguistic foundation and medical professional knowledge, profound translation theory foundation and solid translation skills can also make the translator's translation accurate in content and standardized in language expression. Guided by the definition of functional equivalence, the core concept of Nida's theory, this paper studies the functional equivalence of words, sentences, texts and styles in medical literature translation

Medical science is a highly professional field. Since the reform and opening-up, advanced foreign medical technology and drugs have been introduced into the medical field of our country. In order to promote the development of medical scientific research in our country and shorten the gap between our country and developed countries, researchers and clinicians often need to consult a lot in their work. Documents in the original edition sometimes need to be translated into Chinese for reference at any time. Eugene A. Nida proposed the excellent famous "dynamic equivalence" translation theory, meanwhile, it was named of "functional equivalence". 
As the nature of the translation and taking full consideration of the differences between languages, this paper discusses the functional equivalence of words, sentences, texts and styles in medical literature translation under the guidance of the definition of functional equivalence, which is the core concept of Nida's theory.

\section{Previous Research on Medical English Research Paper Translation}

Functional equivalence is a well-known translation theory proposed by Eugene A. Nida, an American, which has great guiding significance for later translation work. An overwhelming majority of people have made researches and applied this theory in translation practice.

Lu Huan, School of Foreign Languages, Guizhou Normal University, once wrote "Recognition of Nida's Theory of Functional Equivalence", which mainly introduces the theory of functional equivalence. Since it was introduced to China in the early 1980s, it has had a far-reaching impact on Chinese translation circles, but recently this translation theory has been questioned or even totally denied. The author holds that those who disagree with the theory of functional equivalence are due to the absolute understanding of the theory of functional equivalence and a misunderstanding of Nida. The nature as well as principles of the theory of functional equivalence could be discussed. In addition, the proper application of the theory of functional equivalence will be discussed. Obviously, the "functional equivalence" is a dynamic equivalence. Most of the people who oppose "functional equivalence" are because their understanding of "equivalence" is too absolute and they do not really know what Nida is trying to express. Nida once pointed out that "there has never been a situation in which two people are identical in their understanding of language, let alone a completely identical equivalence between the two languages." For translators, they should commit to memory that always shorten the divergence from the source language to the target language, Lu Huan also said in the article, "The content and connotative meaning of the original must be maintained so that the target text can be understood by the reader and cannot be misunderstood. Based on this foundation, the form of information expression ought to be focused on as much as possible."

There are a lot of people who have learned and researched the theory of functional equivalence. Generally speaking, they use this theory to study cases. In China, there are a lot of researchers who are interested in the translation on the literary work and they pay attention to the strategy and skills while translate. In addition, a lot of linguistic studies have been done on the translation of film and variety shows which needs the cultural transformation. What's more, the political translation has been focused on strongly. Many studies have been done on the government work report which could be the key diplomatic channel to the world. Taking this situation into consideration, many translators and professors on linguistic put forward their opinions and did systematic and scientific analysis. There are many researches and discussions, such as Chen Yu's Subtitling Translation of Kung Fu Panda 2 from the Perspective of Functional Equivalence Theory, Shi Chunying's Subtitling Translation of Desperate Housewives from the Perspective of Functional Equivalence Theory, and so on. It is obvious to discover that room for the application of functional equivalence theory is huge.

\section{The Skopos Theory}

The Functional equivalence theory refers to that translation is not in the search of the strict equivalence from the surface lexical. On the contrary, it bears the goal of getting functional equivalence between the source language and target language. In the process of medical translation, in accordance with Nida's theory, translators ought to adopt four points of dynamic equivalence as translation principles to reproduce the meaning of the source language into the target language exactly.

The Skopos theory supports that "the way the target text eventually shapes up is determined to a great extent by the function" (Hatim, 2005: 74). According to the theory of target language, translators need to use the target language. However, there are great differences between language and culture, and there may be great differences between what is translated and what is expressed by the original author. 
According to Vermeer, the audience of the target language is a key factor in determining the use of teleology, and needs vary from country to country and from place to place. This paper emphasizes the purpose of translation activities and the needs of the audience, so that translators have more freedom.

Skopostheorie does not mean equivalence between Skopostheorie and the original text, but it is emphasized that translation should be based on the original expected function. According to different contextual factors, translators should choose the best way to translate. Therefore, teleology has high practicability and scientificity.

Skopos Theory provides more precise and useful translation principles and criteria for the translation of drug instructions. Translation cannot be simply faithful to the original text. Translation is regarded as a cultural transformation between languages. Translators must formulate correct and specific translation strategies according to the characteristics of thetext which should be translated. In the whole process of translation, the translator should consider the differences between different countries in all aspects, so as to achieve the desired translation objectives. Drug names, description, pharmacological, actions, indications, contraindications, dosage and administration, adverse reactions, precautions, package, storage and others are included in the instructions. Translators should try their best to convey accurate Chinese character information to users. Therefore, the translator should abide by the rules that are always audience-oriented and disseminate correct drug use methods.

\section{The Language Features of Medical Papers}

Medical English, as a serious style, is deeply influenced by Latin style. Long sentences have a long history. Long sentences carry rich information in content, and play the role of linking information and emphasizing information in argumentation. A large number of simple sentences with many additional elements and complex sentences with many subordinate clauses are used in medical English literature. Attributive clauses are the most common subordinate clauses. Whereas, long sentences, strict structure and complex composition have been existing, reading must be hard. For example:

Medicare covers a review of your health, education and counseling about preventive services, including certain screenings, shots, and referrals for other care if needed.

a) Studies of nutritional status must take into account the state of the body before and after experiments, as well as the chemical composition of the whole diet and of all material excreted and eliminated from the body (in urine and feces).

b) The modern approach to the problem - after having witnessed the failure of many attempts to solve malnutrition through health interventions alone-is to rather consider malnutrition as the biological translation of a variety of socioeconomic problems afflicting a given society.

\section{Translation Strategies}

As for equivalence of sentence patterns, English and Chinese have great differences in sentence structures. Thus, it is easy to encounter difficulties on this aspect while the translation of long and difficult medical sentences. However, according to functional equivalence theory, when the consistency of content and form between the target language and the source language cannot be realized, the form will be chosen to give way to the content. In the translation of long sentences, the use of functional equivalence theory may disturb the original sentence structure, but the meaning conveyed is the same as that of the original text and can achieve equivalence in content.

An immediate practical problem in dealing with a patient who is thought to have viral meningitides to exclude the presence of leptospirosis or a bacterial infection that might be susceptible to antimicrobial therapy.

In terms of the Skopos theory, it has an absolutely different idea from the functional equivalence theory. It gives priority to the source language and regarding it as the only origin for the communicating information. People who will receive this source language must comply with it 
completely. In addition, skopos theory requires that, on the premise of loyalty to the original text. In order to meet the needs of the target audience, it is necessary to decide which translation method to use - literal translation, free translation or somewhere in between.

Women with fibroid tumors usually have no knowledge of their presence until the tumor gets so large that there is a sense of heaviness in the lower abdomen or until they are conscious of frequent urination from pressure on the bladder or until pressure on the rectum causes obstinate constipation.

\section{Conclusion}

Medical literature must be a key medium for researchers and clinicians to acquire knowledge while working. With the purpose to shorten the gap between China and developed countries, it is in great demand to consult many English medical literatures in the process of work. Seen from a scientific and systematic way, it demands relevant translators to translate into Chinese for reference all the time. Thus, the medical English translation is playing a key role. However, in the process of translation, the handling of many problems, including medical professionalism, vocabulary and word order differences between Chinese and English, have become difficulties in the process of translation. In this paper, it can be seen from the combination of Nida's functional equivalence theory and its examples in medical literature that the functional equivalence theory has guiding significance for the translation of medical literature. Under the guidance of Nida's functional equivalence theory, achieving the maximum equivalence of words, sentences and texts in medical literature translation will be of great benefit to the practice of medical literature translators, medical workers and scientific researchers. Therefore, I think functional equivalence theory should be widely used in the daily translation of medical literature.

\section{Acknowledgements}

This paper is funded by Educational and teaching reform program of Xi'an Medical University (2018JG-48, 2018JG-51); Matching fund program of Xi'an Medical University (2018PT51, 2018PT52); Scientific research program of Department of Education of Shaanxi Province (18JK0650); Innovation and Entrepreneurship Program of Education Department of Shaanxi Province (201825009, 201825003).

\section{References}

[1]. Byram M. Cultural Studies in Foreign Language Education[M]. Clevedon: Multilingual Matters, 1989.

[2]. Littlewood, W. 2011. Communicative language teaching: An expanding concept for a changing world [A]. In E. Hinkel (ed.). Handbook of Research in Second Language Teaching and Learning: Volume II [C]. New York: Routledge. 541-557.

[3]. Marsh, D. 2002. CLIL/EMILE-The European Dimension: Actions, Trends and Foresight Potential [M]. Brussels: European Commission.

[4]. Paul, R., A. Binker, D. Martin \& K. Adamson. 1989. Critical Thinking Handbook: High School [M]. Santa Rosa, CA.: The Center for Critical Thinking and Moral Critique.

[5]. Pavlenko, A. \& J. Lantolf. 2000. Second language learning as participation and the (re)construction of selves [A]. In J. Lantolf (ed.). Sociocultural Theory and Second Language Learning [C]. Oxford: OUP. 155-177.

[6]. Prabhu, N. 1987. Second Language Pedagogy [M]. Oxford: OUP. Stoller, F. \& W. Grabe. 1997. The six-T's approach to content-based instruction [A]. In M. Snow \& M. Brinton (eds.). The Content-based Classroom: Perspectives on Integrating Language and Content [C]. New York: Longman. 78-94. 